\title{
A Review of Cognitive Radio Smart Grid Communication Infrastructure Systems
}

\author{
Daisy Nkele Molokomme ${ }^{1}\left(\mathbb{D}\right.$, Chabalala S. Chabalala ${ }^{2, *}$ (D) and Pitshou N. Bokoro ${ }^{1(\mathbb{C})}$ \\ 1 Department of Electrical and Electronic Engineering Technology, University of Johannesburg, \\ Johannesburg 2028, South Africa; 201132400@student.uj.ac.za (D.N.M.); pitshoub@uj.ac.za (P.N.B.) \\ 2 School of Electrical and Information Engineering, University of the Witwatersrand, \\ Johannesburg 2050, South Africa \\ * Correspondence: chabalala.chabalala@wits.ac.za
}

Received: 10 May 2020; Accepted: 16 June 2020; Published: 23 June 2020

\begin{abstract}
The cognitive smart grid (SG) communication paradigm aims to mitigate quality of service (QoS) issues in obsolete communication architecture associated with the conventional electrical grid. This paradigm entails the integration of advanced information and communication technologies (ICTs) into power grids, enabling a two-way flow of information. However, due to the exponential increase in wireless applications and services, also driven by the deployment of the Internet of Things (IoT) smart devices, SG communication systems are expected to handle large volumes of data. As a result, the operation of SG networks is confronted with the major challenge of managing and processing data in a reliable and secure manner. The existing works in the literature proposed architectures with the objective to mitigate the underlying QoS issues such as latency, bandwidth, data congestion, energy efficiency, etc. In addition, a variety of communication technologies have been analyzed for their capacity to support stringent QoS requirements for diverse SGs environments. This notwithstanding, a standard architecture designed to mitigate the aforementioned issues for SG networks remains a work-in-progress. The main objective of this paper is to investigate the emerging technologies such as cognitive radio networks (CRNs) as part of the Fifth-Generation (5G) mobile technology for reliable communication in SG networks. Furthermore, a hybrid architecture based on the combination of fog computing and cloud computing is proposed. In this architecture, real-time latency-sensitive information is given high priority, with fog edge based servers deployed in close proximity to home area networks (HANs) for preprocessing and analyzing of information collected from smart IoT devices. In comparison to the recent works in the literature, which are mainly based on CRNs and 5G separately, the proposed architecture in this paper incorporates the combination of CRNs and $5 \mathrm{G}$ for reliable and efficient communication in SG networks.
\end{abstract}

Keywords: cognitive radio (CR); cloud computing; Fifth-Generation (5G); fog computing; information and communication technologies (ICTs); smart grids (SGs)

\section{Introduction}

Traditional power grids have been designed to fulfill the 20th Century needs of consumers. These power grids consist of four major domains: generation, transmission, distribution, and consumption. In this configuration, information and communication technology (ICT) and intelligence (cognitive) features, if any, are usually found within the high-voltage domain $[1,2]$. It was argued in [3] that existing grids could be considered as a mere power transportation from a few central power plants to numerous consumers. These grids are prone to power disturbances and unplanned outages, electricity theft, as well as inaccurate electricity metering and billing [4]. These shortcomings stem from the obsolete communication infrastructure and outdated technologies 
continuing to be relied upon to carry out the primary function of power grids [5]. Furthermore, the existing or traditional power grids are known for their characteristic feature of a one-way flow of information and electricity [6-8]. This implies that centralized generating plants (e.g., combined heat and power (CHP), hydro plants, etc.) can only distribute power to consumers through traditional infrastructure [9]. Despite their shortcomings, traditional power grids have revolutionized the daily lives of consumers since their inception. Fundamentally, the existing power grids suffer from a lack of pervasive and effective real-time communication between consumers and utility control center (UCC), which yields a poor quality of experience (QoE) for consumers [10]. A typical traditional power grid is depicted in Figure 1. Among these deficiencies, one of the most significant is the aging communication infrastructure, which comprises electromechanical metering, manual restoration, and limited monitoring abilities [8]. In essence, traditional power grids were designed without the consideration of 21st Century challenges [5]. To resolve the aforementioned issues, the smart grid (SG) concept has been developed towards the realization of an effective and reliable solution. The concept of SG has been around for several years. However, due to the evolution of advanced ICT, SGs gained significant attention in the academia and research industry recently. In essence, SGs comprise the incorporation of advanced ICT and functionalities intending to mitigate existing grids' challenges [11]. ICTs are envisaged as an essential requirement for sensing, analyzing, monitoring, and integration of core elements into the network architecture [12,13]. Hence, the design and practical implementation of an efficient ICT infrastructure should be considered as a critical task towards the realization of SGs [2]. Prior works have therefore mainly focused on a variety of appropriate ICT architecture designs and frameworks in order to address the unique and diverse QoS requirements for SGs [14-21].

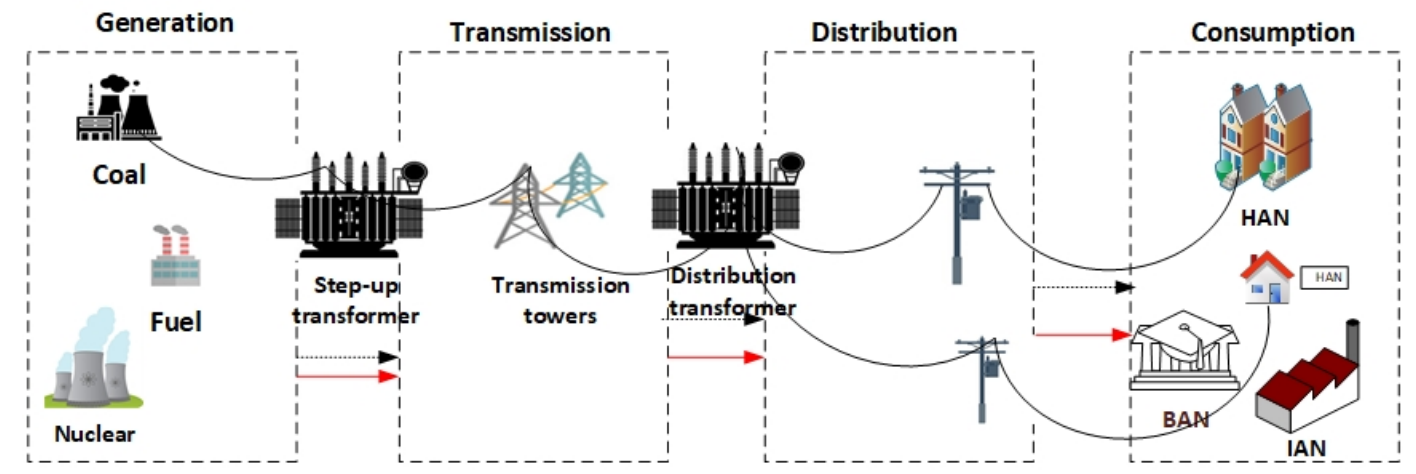

Figure 1. Conceptual model of traditional power grids. HAN, home area network; BAN, business area network; IAN, industrial area network.

The development and practical implementation of SGs mandate a complete redefinition of power grids phenomenon from radial to network architecture, with the exploitation of the two-way flow of information and electricity [22]. However, this paradigm emerges along with various challenges that consist of distinct and stringent QoS requirements to be fulfilled, such as latency, security, reliability, and efficiency, to mention a few [23,24]. Some of the emerging concepts associated with SGs include distribution automation (DA), demand response (DR) [25], wide-area monitoring systems (WAMS), demand side management (DSM) [26], and advanced metering infrastructure (AMI) $[27,28]$. To support the QoS requirements, the following enabling technologies must be developed and implemented: (1) ICTs to facilitate an open architecture for plug-and-play smart appliances, (2) two-way communication technologies to enable interoperability among SGs components, and (3) software and hardware interfaces to ensure greater control optimization and monitoring [29,30]. With no strategic path developed to implement SGs yet, several works for pilot projects such as the qatar general electricity and water corporation (KAHRAMAA)AMI pilot research project have been used as a guideline towards the realization of SGs [31-33]. Some of the previous works proposed the integration of various communication technologies to mitigate QoS issues in SG networks. 
Besides their demanding QoS requirements, SGs promise to transform the intelligence level for all stakeholders at different levels. This can be achieved by the facilitation of two-way communication, increased integration of distributed generation (DG), and storage technologies, as well as improved interoperability among the electricity supply industry (ESI) and customers [15]. The aforementioned benefits are thoroughly discussed in subsequent sections. With the increasing demand for wireless applications and deployment of smart IoT devices, latency and security are among the major issues in SGs' communication architecture [34]. As a result, security has been one of the main focuses of research in SGs [20]. Furthermore, wireless communication architecture based on wireless mesh networks (WMN) was proposed in [20]. However, large-scale deployment of SG components based on WMN may not be an efficient solution due to issues related to latency, complexity, and high power consumption. In terms of complexity, each node in WMN is considered as a gateway. As a result, data packets need to be transmitted through each hop to reach their destination, which results in communication delay. Alternatively, this paper proposes a single-hop architecture based on 5G and CRNs, with the aim to improve reliability, as well as spectrum efficiency and energy efficiency by adopting some of the ideas presented in $[35,36]$. This also involves investigation on how to employ unused spectrum bands efficiently $[37,38]$. In general, the main components of SGs include the large-scale deployment of smart devices, sensors, and UCC with smart meters (SMs) serving as a gateway [39]. In order to allow remote and advanced control services within grids, these components are usually connected to the cloud servers via the Internet backhaul network [40]. Traditional SGs adopt cloud services for data storage, processing, analysis, and decision-making. Since cloud servers do not possess features such as scalability, edge analytics, real-time interactions, and service delivery, they fail to manage the huge data volume. For instance, cloud servers are geographically distributed. Thus, consumers and power providers may fail to access some services from the cloud. Moreover, cloud servers are not latency-sensitive for real-time applications. This results in huge delays and packet losses. Inherently, security and privacy are major challenges in cloud servers since they operate on shared backgrounds (public servers) [41]. The IoT smart devices generate raw data transmitted to UCC periodically to cloud servers. Such data may contain personal information of consumers, which if compromised may place the safety of consumers at risk. However, with the evolution of advanced technologies, fog computing has been identified as an effective technique to resolve the issues in traditional cloud servers. Fog computing can be defined as an extension of cloud servers by incorporating essential features that cloud servers do not have. This includes the ability to support latency-sensitive and real-time communication, location awareness for IoT devices in home area networks (HANs), analyzing data without the need to be transmitted to the cloud, and the ability to manage a huge volume of data. This paper aims to employ the combination of fog computing and cloud computing to address latency and security issues. Considering a large number of smart devices and wireless applications, the deployment of ICT infrastructure is a critical design issue in SGs. This paper also presents the review of the state-of-the-art architectures and the underlying issues associated with the design and implementation of ICT infrastructure in the context of SGs. The key contributions of this paper are outlined as follows:

- To provide an extensive review on the state-of-the-art architectures proposed in the literature and to fulfill the distinct and stringent QoS requirements in SG communication systems.

- The incorporation of fog computing and cloud computing with the aim to support QoS requirements for SG communication systems such as latency and security.

- The identification of possible communication technologies that can mitigate QoS requirement issues such as spectrum efficiency, energy efficiency, and security as latency.

- To propose a hybrid SG communication architecture based on CRNs and 5G mobile technology, with the aim to improve reliability and network efficiency.

The rest of this paper is organized as follows: Section 2 presents the review on the state-of-the-art architectures from the existing works. Section 3 presents the next-generation power grids and the active players for the realization of SGs. In Section 4, an overview of hierarchical communication layers 
for SGs is explored. Then, Section 5 presents network communication requirements and technologies, followed by Section 6 for the proposed architecture. Finally, the paper is summarized in Section 7.

\section{Related Works}

The significant increase in SGs wireless applications and smart IoT devices yields rapid growth in the vulnerability of network stability and data privacy. SGs encounter the challenge to optimize network configuration intelligently and issue control demands autonomously without the intervention of a third party. The authors in [42] reported a significant innovation that ICT brought into major domains of grids. The rise in data volume becomes a challenging task for the communication architecture to manage and process in a reliable manner. As a result, prior works have devoted significant attention to enhancing QoS in SG networks, which entails finding the appropriate communication technologies to mitigate latency, response time, and security issues, among others [40]. This section presents the related prior works, subdivided into two categories.

\subsection{Traditional Architectures}

To resolve QoS issues in SGs, prior works proposed several types of ICT architectures, each of which had different research directions and objectives. The work in [42] was among the initial works that surveyed energy-efficient SG communication infrastructures, with the objective of improving energy efficiency. In [7], the authors outlined vital aspects in the deployment of SGs including standard interoperability, SG applications, and communication technologies, both wired and wireless. A similar study that reviewed issues related to SG communications architecture was also presented in [29]. Furthermore, the authors in [7,43] identified issues in designing and implementing SGs, which included: energy efficiency, the instable nature of DG, interoperability, and security. The incorporation of DGs in SGs is one of the emerging techniques aspiring to enhance performance in SG networks. However, the instable nature of wind and solar systems in DGs affects the overall performance of the network, especially if mismanaged [44]. Despite their demerits, DGs mainly contribute to enhancing the resiliency of SG networks and reducing the energy bill of customers. Improving the reliability of DSM is among the major research focus areas in SG deployments. An optimization problem regarding the energy storage system that analyzed the impact of battery size and operational decisions on peak shaving technique was presented in [45]. Peak shaving involves the flexibility of consumers in the daily operational grid, by allowing customers to purchase from and sell electricity to the grids. This yields improved energy efficiency and stability of the power grids. With the significant increase in wireless applications and multiple transmission points, conventional architecture finds it difficult to manage and control data traffic in the network. To control, monitor, analyze, and optimize the complex and dynamic SG environment, reliable and secure infrastructure is of great importance [13]. However, several aspects of SGs need to be investigated and established towards the design of appropriate communication infrastructure [46,47]. The authors in [43] conducted a survey on existing technologies using the wired and wireless medium to address a variety of issues in diverse SG environments. Furthermore, SGs are expected to employ more than one technology due to their dynamic nature. In essence, the design and implementation of SG infrastructure is a challenging task. To enable communication between HAN and wide area network (WAN), the AMI architecture has been proposed [48]. In this architecture, the IEEE 802.11/IEEE 802.15.4 standards are considered to be ideal for short- and medium-range communication (i.e., from SMs to SMs and SMs to gateways). AMI has been identified as an initial step in the realization of SGs [49]. An indoor architecture design was developed in [3]. In the development of their proposed architecture, three aspects were investigated: advanced meter reading (AMR), advanced meter management, and AMI [50]. In this model, the focus was solely placed on improving the energy efficiency and reliability, which were reported to have been achieved. However, the communication technology employed was not mentioned. The development of suitable architectures is mandatory as the existing technologies may not be able to fulfill the diverse and stringent QoS requirements for SGs. 


\subsection{The State-of-the-Art Architectures}

The data generated in SGs require a reliable infrastructure for transmission in a secure and scalable manner. In the case of wireless networks, connectivity and resource optimization are one of the key factors that affect network performance [51]. In addition, the issue of packet loss due to fading channels is inevitable in wireless networks [52]. Fourth-Generation (4G) based communication infrastructure was proposed in [53]. The authors highlighted issues related to the adoption of $4 \mathrm{G}$ technology in a diverse SG environment, taking into consideration the demanding QoS requirements. Among other issues, the dynamic structure of $4 \mathrm{G}$ has been highlighted as a challenge for its adoption in ubiquitous SG applications. With the emergence of advanced mobile technologies, 5G has been realized as an effective technology for diverse the SG environment. In [13], a comparison between the 4G Long-Term Evolution (LTE) and 5G technologies was conducted for power network monitoring and control, with the aim to evaluate the performance of SG distribution networks under fault management. In [54], the authors presented a comprehensive review on 5G and its level of support for SGs' deployment. However, their focus was solely placed on enhancing energy efficiency by adopting 5G. Several aspects were highlighted in this work, which included the challenges of adopting 5G in the SG environment. Among the challenges highlighted were standard interoperability and data congestion due to the significant increase in the deployment of smart IoT devices. An increase in power consumption due to smart IoT devices may be a challenging issue in their proposed architecture. To address the issue of power consumption, CRNs were considered in [36]. In [55], a CRN communication infrastructure was proposed. The proposed infrastructure was divided into three hierarchical layers: cognitive HAN, neighborhood area network (NAN), and WAN. The main aim of their proposed model was to enhance throughput and dynamic spectrum access and minimizing interference between the users. Currently, wireless technologies are the most preferred over wired technologies in SGs, due to their robustness, reliability, and less installation and maintenance costs, among other benefits as discussed in detail in Section 5. With the evolution of advanced technologies, 5G is a prominent technology to mitigate the underlying issues. In [56], the authors proposed a CRN based communication architecture to address the traffic issue within HAN/WAN frameworks. To improve the resilience of the network and allocation of spectrum bands, the proposed framework was developed to control data traffic between licensed and unlicensed users. This was achieved by identifying the available spectrum, as well as defining the state of licensed users [57]. The prior works proposed architectures based on 5G and CRNs separately. This paper proposes an architecture based on the joint operation of $5 \mathrm{G}$ and CRNs in order to improve the reliability and efficiency of networks.

\section{The Next-Generation Power Grid}

The next-generation power grids consist of a variety of essential components distributed in wide geographical areas. A conceptual model of the National Institute of Standards and Technology (NIST) is an ideal definition of SGs' operation. This model was designed to support the network planning, requirements, and development of ubiquitous SGs with distinct QoS requirements, as depicted in Figure 2.

It consists of seven major domains: electricity market, network operation, service provider, and power systems (e.g., bulk generation, transmission, distribution, and consumption). With an exponential increase in mobile users in the next-generation power grid, spectrum and energy efficiency are among the major challenges in the deployment of the new paradigm. In order to allow interoperability across different domains, reliable and efficient communication technologies need to be incorporated within the SG infrastructure, with the ability to meet the QoS requirements for each of the stakeholders involved. To address the fundamental issue based on finding an appropriate technology for SGs' realization, this section presents an overview based on the communication requirements, as well as the existing and emerging technologies. Some of the previous works in the literature surveyed wireless and wired communication technologies with the potential to address the varying 
QoS requirements for SGs [58]. Some of the investigated technologies are presented in Section 4 of this paper. The next subsections describe the roles of different domains for the next-generation power grid.

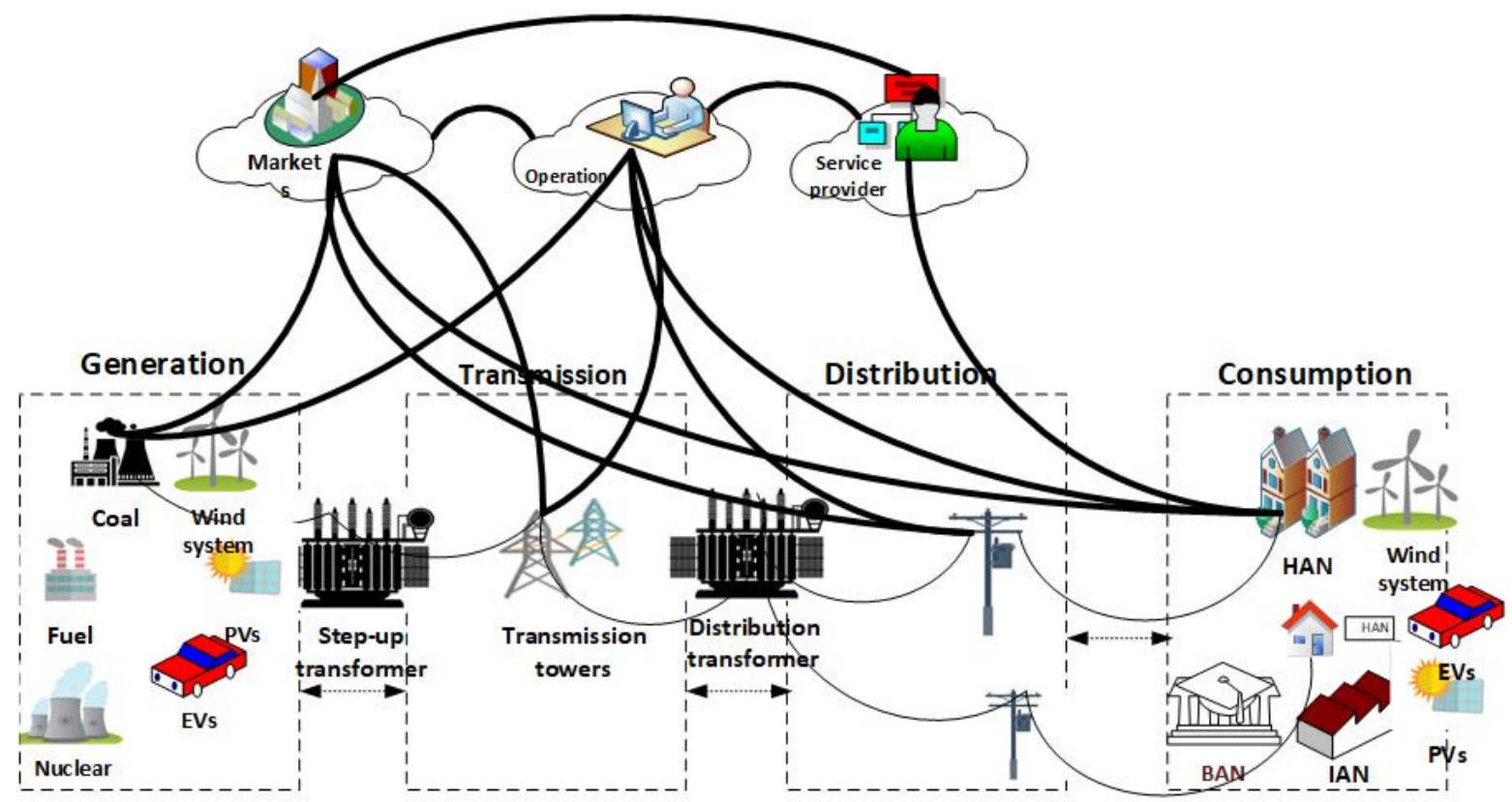

Figure 2. NIST conceptual model for smart grids [24].

\subsection{Bulk Generation Domain}

Traditionally, the power generation grid is associated with the adoption of thermal, nuclear, and hydro plants to generate electricity. In the next-generation power grid, renewable energy sources (RESs) promise to take over for the fossil fuels. The adoption of RESs such as wind and solar systems in the generation grid aims to minimize the carbon footprints in terms of the environmental sustainability index (ESI). Despite their benefits, an increase in the penetration of RESs into the generation power grids further complicates issues due to their instable nature [59]. In order to resolve the issue of instability, the concept of DG has been developed. DG can be defined as an integration of various power sources and storage technologies in the vicinity of the consumer's premises and power generation domain. This comprises the integration of RESs with advanced communication technologies and storage technologies such as plug-in electric vehicles (PHEV) [60]. The enhancement of energy efficiency and reliability using these techniques will be achieved by supplying energy into the macro grid when renewable energy production is low or simultaneously storing energy when renewable power production is high. This technique is known as vehicle-to-grid (V2G)/grid-to-vehicle(G2V), respectively. Previous research focused on the issue of managing the DGs as a result of instability and variations in geographical deployments. To address the above-mentioned issues, virtual power plants (VPPs) have been identified as a viable solution. VPP can be defined as an aggregation of DGs into a single entity; thus improving the stability and reliability of power grids. Moreover, the issue of dispatchability affects the stability of RESs. In [61], a criterion to address dispatchability in DGs was proposed, where the authors also presented the analysis of RESs with the objective to enhance energy efficiency.

\subsection{Transmission Domain}

The transmission grid has played an essential role in the delivery of power to consumers in an efficient, reliable, and secure manner in the existing power grids and will continue to ensure a high degree of continuity of power supply in future power grids [5,62]. With the significant increase of advanced ICTs and power electronics components in SGs, an overall performance and intelligence 
feature of the transmission grid is transformed to high level. This is due to enabling technologies such as flexible AC transmission systems (FACTS), optimized transmission dispatch, high capacity conductors, and advanced storage systems, among others, which offer a great solution to enhance the network capacity of the transmission grid [5]. The primary function of the transmission grid in ESI is to maintain the quality of electricity over long distances to consumers, while also serving as a communication bridge between remote generating plants and consumers. In order to realize the benefits that emerge with these enabling technologies, the objectives of the transmission grid need to align with that of SGs. However, the traditional transmission grid was not designed to cater to some concepts that emerge with SGs' innovation. These include ensuring interoperability between energy markets and consumers, significant variation in the utilization of transmission, and instability complications that arise with the penetration of RESs such as wind and solar systems [63]. In a nutshell, the smart transmission grid aspires to enable constant reconfiguration of power generation sources depending on power demand and availability [64].

\subsection{Distribution Domain}

The design of distribution systems in conventional grids is dependent on the radial design architecture, with centralized generation, limited sensors, manual monitoring, and restoration abilities [65]. In this sense, a low degree of the distribution network in the existing grid is transformed into a smart distribution by facilitating sensors throughout the network. As a result, this improves the restoration and optimization control abilities of the network in the case of unplanned outages and scheduled interruptions. Therefore, this leads to an advanced concept of a distribution management system, which will be achieved through a decentralized network paradigm that incorporates energy management incentive programs to balance the load demand and supply [66]. The SGs encompass advanced ICT infrastructure in the network, which guarantees an improved automatic restoration and pervasive control of real-time communication in the network [67]. This leads to the fundamental question of determining a candidate communication technology to ensure the realization of these benefits in the future distribution network. A smart distribution network addresses some stringent requirements such as high throughput and reliability. This offers efficient infrastructure for information transmission, interoperability, and allowing real-time communication. Discontinuous transmission is also identified as an essential technology to improve energy efficiency, particularly in wireless cellular networks [68]. This is achieved by incorporating the sleep/active mode technique on particular components embedded in the network, which further minimizes the power consumption and improves the reliability of the grids.

\subsection{Electricity Markets}

The conceptual model depicted in Figure 2 above consists of actors or domains that are distinguished according to their functions and responsibilities in an SG architecture. In this sense, electricity markets consist of actors involved in electricity trading, providing control commands, or incentive programs, which aim to manage and balance power demand and supply. These include power providers, customers, traders, and aggregators [24]. The information collected from this domain can further be used to profile customers for specific and targeted advertisements.

\section{An Overview of Communication Infrastructure}

A typical communication infrastructure for advanced metering is depicted in Figure 3, which illustrates an ideal definition of the two-way flow of communication concept. This infrastructure comprises three hierarchical communication layers: WAN, NAN, and HAN. 


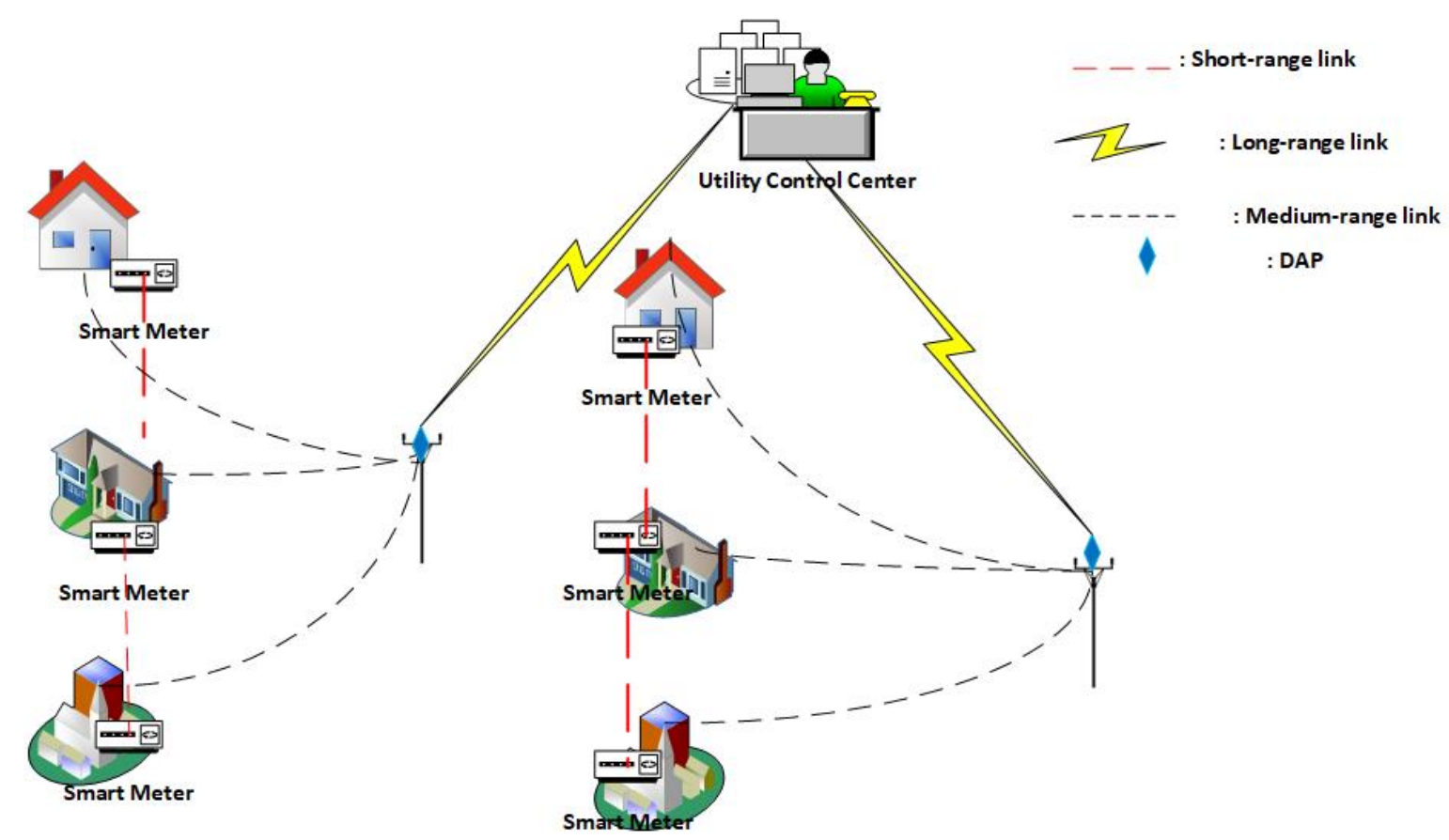

Figure 3. A typical two-way communication architecture.

\subsection{Wide Area Network}

Conventionally, WAN comprises a backhaul network with cloud servers geographically distributed over the network. In SGs, WANs facilitate communication between the electricity markets and power providers. To enable communication within the diverse SG network, WAN serves as a communication link between UCC and HAN. Furthermore, WAN links these servers to UCC to enable communication among them. 5G can be considered as an ideal technology for WAN applications and services. The CRNs can also be adapted to improve the spectral efficiency of the network by dynamically accessing unused spectrum bands [69].

\subsection{Neighborhood Area Network}

The primary function of NAN in SGs is to serve as a communication bridge between HAN and WAN; that is, transmission of real-time power consumption data of each smart electrical appliances embedded in HAN. NANs interconnect HANs and also serve as the communication link between HANs and WANs. NANs serve as an intermediary node, by transmitting SMs' information to UCC. The selection of communication technologies in this layer varies due to the SG applications. Hence, both wireless and wired communication technologies are ideal solutions for NAN applications with diverse QoS requirements. Optical fiber and 5G are considered ideal technologies for NAN applications due to the ability to support real-time latency-sensitive applications. Furthermore, implementing a suitable information architecture between smart meters, sensors, and utility data centers is still a challenge. As a result, NAN gateways need to be deployed to transmit information from heterogeneous IoT smart devices to WAN [70]. In [71], the authors reported the adoption of wireless communication technologies as the appropriate solutions for NAN applications due to their ability to enable rapid deployment in a cost- and energy-efficient manner.

\subsection{Home Area Network}

The customer's premises area network can be categorized into three depending on the environment: HAN, business area network (BAN), and an industrial area network (IAN) [18]. These areas can be distinguished by climate and economic conditions, among other factors. The load demand in HAN is determined by the daily routine of the consumers integrated into the network, 
as well as the change in climate conditions. The load demand from consumers during peak hours and winter season is expected to be higher than during sunny seasons and off-peak hours; whereas in BAN, the load demand is determined by the economic condition and is less impacted by the change in climate conditions. Hence, HAN serves as a communication link between smart devices and SMs. The primary function of SMs in HAN is to collect power consumption data and monitoring the grid status in real-time. The traditional communication architecture fails to manage the huge data volume generated from SMs. AMI has been realized as the paradigm to support QoS requirements in HAN [72,73]. Furthermore, AMI enables two-way communication between smart devices and SMs. The DR and DSM are among the concepts that emerge with the innovation of AMI to enhance the reliability and stability of grids. The DR concept can be achieved by employing advanced communication technologies that will enable electric utilities to turn on/off smart power plugs wirelessly within consumer's households based on the on/off-peak periods [74]. However, finding a suitable communication technology is still a challenge. Several works have proposed power line communication (PLC) as the candidate technology, but there is still a debate on the role of PLC in SGs. HAN applications require a coverage area ranging up to $100 \mathrm{~m}$, tolerable latency, and also support low bandwidth within the smart meters and electrical appliances. ZigBee, Wi-Fi, HomePlug, Z-Wave, and M-Bus are identified by the Association of Home Appliance Manufacturers (AHAM) as the best candidate technologies for HAN applications [29].

\subsection{Cognitive Radio Network in SGs}

Two-way communication is imposed as an essential fundamental for the realization of SGs. In this manner, SGs comprise multiple transmission points, as well as an increase in mobile users, which leads to data congestion and packet losses. This further minimizes the reliability and concurrently maximizes energy consumption in the network. The demand for radio spectrum increases, leading to a scarcity of radio resources in the wireless network. The adoption of CRNs has been realized as an effective solution for inefficient use of the spectrum $[69,75]$. In the presence of CRN, the HAN gateway enables a bi-directional flow of communication between the smart devices deployed within the customer's premises. Furthermore, the gateway provides an application of cognition capability to enable SGs to adapt to changing environments [76]. For management purposes, the collected data are further transmitted to UCC to initiate necessary commands such as dynamic incentive programs. Concurrently, IoT smart devices within HAN allow consumers to manage their power consumption. As a result, the cognitive radio (CR) NAN gateway reduces data traffic on the network by detecting unused frequencies on the network. Furthermore, NAN gateways allocate IP addresses and channels to all smart devices deployed in HAN; thus reducing data traffic on the network. In other words, CRNs allow two radio users, i.e., primary user (PU) and secondary user (SU), to share the same frequency band $[36,57]$. This is achieved by allowing the SU to utilize the licensed spectrum opportunistically in the absence of PU. The adoption of CRN in SGs might improve the reliability of the network and reduce data traffic by improving the utilization of unused spectrum [4]. However, to improve data traffic between SU and PU, CRN needs to sense the state of PU before allowing SU to utilize a certain spectrum. PU can either be in active or the idle state as defined in [77]. Prior works have proposed unique schemes to manage and reduce communication interference between PU and SU in CRNs [36,78].

\subsection{Issues in Traditional Cloud Servers}

Traditional SGs comprise cloud servers for data storage, processing, and analysis [79]. This architecture design is prone to security challenges, communication delay, and stability issues. The main components include SMs, gateways, backhaul network, and cloud control center, as depicted in Figure 4 with reference to [24]. SMs gather information periodically from smart IoT devices in HAN and transmit it to the control center for further decision-making [39]. However, since SMs are located far away from the control center, gateways are deployed to aggregate and transmit the data in manageable sizes to the control center. In this decentralized network, distributed cloud servers form a 
multi-hop network. Therefore, during transmission of data, each packet needs to pass through multiple hops deployed on the network before it can reach its final destination (control center), which leads to the rise in communication delays and packet losses.

The significant increase of IoT functionalities in SGs results in a huge data volume, which also leads to data congestion since traditional cloud servers fail to manage the huge data volume. Furthermore, cloud based architectures operate on shared backgrounds (public) [80,81], which increases the vulnerability and safety of consumers.

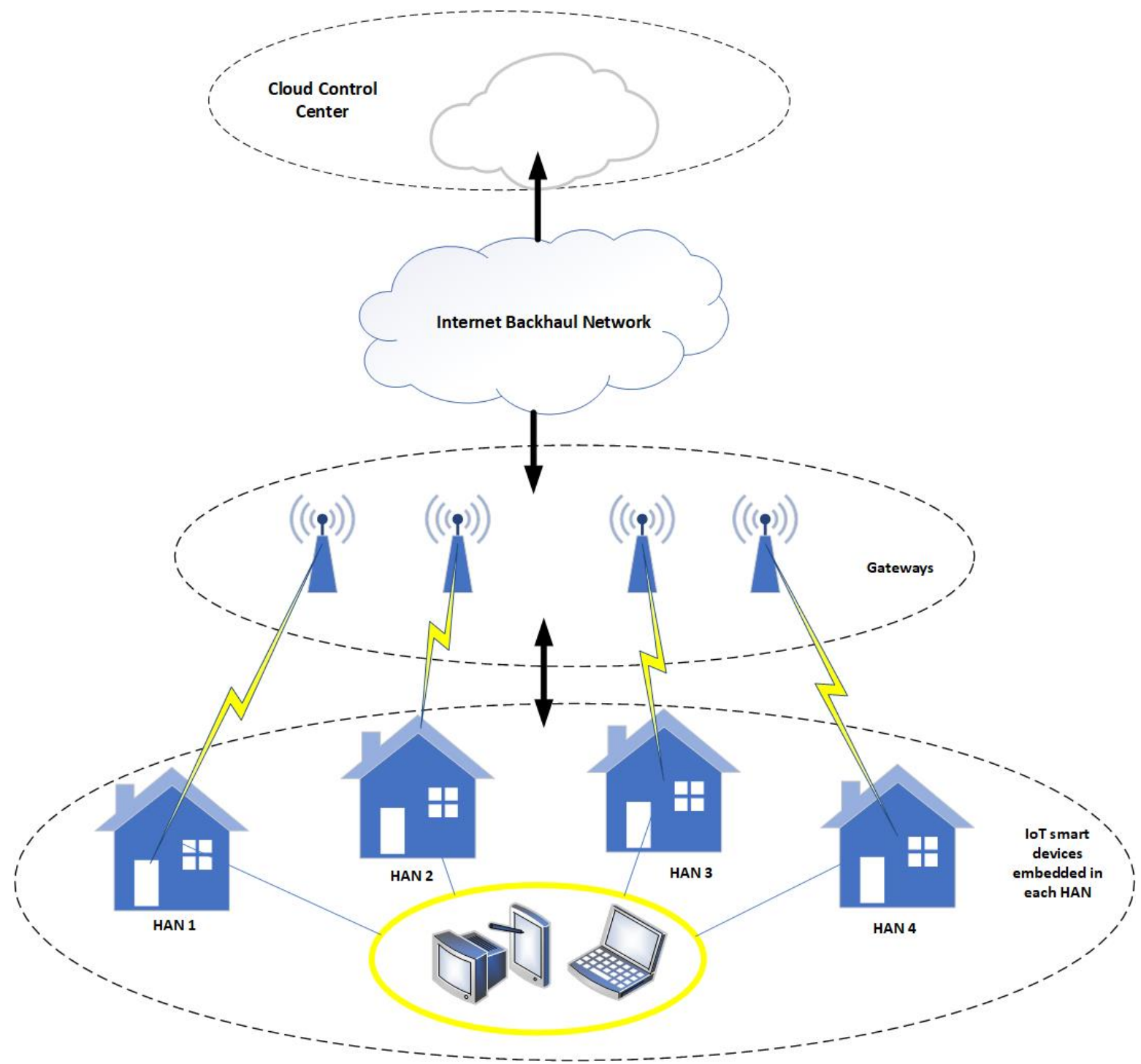

Figure 4. Traditional cloud architecture.

\subsection{Fog Computing as a Solution}

In 2012, Computer Information System Company (Cisco) identified the fog computing concept as a solution to address issues in cloud computing. Fog computing is not a replacement of cloud computing, but an extension by offering additional features to traditional SGs [82]. Additionally, this concept is considered as an essential requirement in 5G. Fog nodes serve as the intermediary between SGs and cloud servers [83]. To manage and analyze the large data volume, a fog computing architecture such as illustrated in [40] is proposed in this paper. The proposed architecture comprises a group of fog gateways that transmit raw data to the servers for further preprocessing and analytic purposes. However, since fog servers are located close to HAN, data can be analyzed in fog servers without the intervention of any third parties. Furthermore, fog computing manages smart devices 
as different clusters/classes based on a certain criterion to reduce data traffic. To reduce traffic in the network, fog gateways receive merged data from their associated clusters. The distinguishing factor between different clusters is explained in detail in Section 6 of this paper. The data are further transmitted to cloud servers depending on their priority classes.

\section{Communication Network}

In SGs, communication infrastructure has become an essential element towards addressing the requirements of power grids by incorporation of advanced ICTs and intelligence features [84]. Different communication requirements for SG networks are discussed in the sequel.

\subsection{Communication Requirements}

\subsubsection{Latency}

The information processed within SG applications and services is distinguished by its latency sensitivity. For instance, communication delay in non-real-time communication can be tolerable, whereas in the case of real-time communication, such as fault detection, power or service restoration, quality monitoring, etc., the level of latency sensitivity is extremely high. In [47], latency was defined as the time between when the state occurred and when it was acted upon by the application (delay). However, in a diverse SG environment, the latency requirements for different applications vary.

\subsubsection{Bandwidth}

The variety of wireless applications and services employed in SGs consist of distinct levels of bandwidth requirements, which range from low, medium, to high radio frequency ranges. In small-scale SG applications such as AMI and DR, the low to medium radio frequency should be sufficient for data delivery in the network, whereas in large-scale applications such as virtual power plants (VPPs)and meter data management system (MDMS), medium to high radio frequencies are required. In essence, bandwidth can be defined as a range of frequencies required for the transmission of data for various applications.

\subsubsection{Security}

Security is the ability of the communication infrastructure to combat physical and cyber-security attacks to protect the critical data gathered from various smart grid components. SG applications and services consist of crucial information, which if accessed and/or leaked by unauthorized person, can be utilized to break the stability of the grid and/or to access the daily routines of consumers. Information leakage can occur during data transmission or storage [85]. Traditional power grids utilize electromechanical meters installed on customer's premises to measure, gather, and store power consumption data. However, this system poses several concerns or issues to both utilities and customers, such as the possibility of meter reading errors, i.e., the under-billing or over-billing of customers since manpower is hired to acquire data from the consumer's premises manually. To mitigate security issues in SGs, HAN and SMs are deployed on the customer's premises.

\subsubsection{Reliability}

Reliability can be evaluated as the probability of successfully transmitting a specified number of bytes within a certain user-plane latency, given a certain channel quality, e.g., a few SMs, or coverage-edge [86]. To ensure accurate continuity of communication between devices, a reliable communication architecture is required. Reliability is considered as the basic requirement of communication networks in SGs, which determines the availability of data transmission links [87]. In traditional power grids, the detection of power outages on time is still a challenge due to a lack of automated analysis and few sensors deployed in the network. The concept of load-shedding has been 
another technique to improve the system reliability to an acceptable level by reducing the load when all the available generation units are exhausted [88]. In SGs, features such as smart load control (direct or indirect) are allowed to improve the reliability and QoS of the network.

\subsection{Communication Technologies}

\subsubsection{Power Line Communication}

In this technology, information is transmitted through existing transmission lines at a rate of $2-3 \mathrm{Mb} / \mathrm{s}$, which results in low installation cost. PLC is suitable for HAN applications due to its characteristic features of low data rate, low bandwidth, etc. [27]. Furthermore, it is usually applied in remote metering and home automation applications. However, the authors in [8] reported that the debate regarding the application of PLC in SGs is still an open issue. Signal attenuation is a major drawback in PLC due to the operating nature of transformers [7]. As indicated in [64], some of the challenges for PLC include the inability to transmit data over long distances and the noise level generated by power lines. Considering the increasing amount of data that need to be transmitted over the same medium for different applications, the massive data volume remains a challenge that may be beyond the capacity of PLC systems.

\subsubsection{Optical Fiber}

Optical fiber is considered as the appropriate wired communication technology to be implemented for various WAN applications, due to its ability to withstand the noise levels and also transmit the information over long distances. Optical fiber can be an ideal communication technology for SG applications such as distribution automation, DR, AMI, etc. [89]. Optical fiber provides several benefits such as high bandwidth, immunity to radio interference, and its ability to eliminate virtually unauthorized access of information such as eavesdropping, which inherently improves network security. Despite its benefits, it poses some disadvantages such as high installation costs due to the need to map them along with the existing distribution and transmission network lines, limitations in terms of geographical areas that may be difficult to reach, and the overall time it would take for practical implementation.

\subsubsection{ZigBee}

ZigBee is a wireless technology that is designed for radio frequency applications that require a low data rate, a long battery life, and secure networking and is also based on the IEEE 802.15.4 standard $[8,90]$. This technology is ideal for short-range communication such as HAN applications. SGs comprise a variety of wireless applications and IoT smart devices with distinct QoS requirements. Considering the huge data volume generated from these components, ZigBee may not be appropriate for latency-sensitive applications such as remote monitoring and distribution automation because of its inability to share the same communication medium with other applications, as well as low processing capabilities.

\subsubsection{Wireless Cellular Technologies}

In recent years, LTE also known as 4G technology has been making waves in the industry as the promising technology for crucial applications such as smart homes, health care, etc. The traditional 4G already provides enhanced mobile broadband (eMBB) for its applications. As compared to other existing technologies, $4 \mathrm{G}$ is the most preferred technology in most cases due to low installation and maintenance costs, as well as its ability to transmit large volumes of data over long distances. $4 \mathrm{G}$ presents some features that were not used before in wireless and mobile technologies [53]. 4G's benefits include deployment flexibility, high data ratio, and efficient capacity for SG applications and services. However, security remains a major challenge in $4 \mathrm{G}$ since it operates on shared backgrounds. Initially, $4 \mathrm{G}$ was not designed to cater to some of the SG applications; therefore, its adoption on ubiquitous SGs becomes a challenging issue [91]. 
Considering the information generated by smart IoT devices and sensors, reliable technology with the ability to ensure the privacy of such information is required. Prior works such as in [32] proposed the use of $4 \mathrm{G}$ in SGs' deployment. With the evolution of advanced ICTs, $5 \mathrm{G}$ has recently been gaining popularity or preference over other wireless technologies [92]. In [35,93], the authors defined 5G as the revolutionizing mobile communication providing a pervasive and ultra-broadband fiber-like experience for everyone and everything to consume emerging mobile services. These services include but are not limited to three-dimensional or ultra-high-definition video sharing, machine type communication (MTC), intelligent transportation systems (ITS), and smart homes. The 5G framework comprises both the unlicensed and licensed frequency bands, which can be exploited to enhance spectrum and energy efficiency [54]. A set of techniques developed in 5G includes massive machine type communication (mMTC), ultra-reliable and low latency communications (URLLC), and eMBB, which is currently offered in 4G [94]. Considering that SGs consist of large-scale deployment of SMs and sensors, URLLC may be an effective service to offer support for latency-sensitive applications in SGs. Since SGs are among the main applications of 5G, this can be an appropriate technology for real-time latency-sensitive applications and services.

\section{Proposed Cognitive ICT Infrastructure}

The conceptual model is depicted in Figure 2, which adopts communication technologies belonging to the IEEE 802.11 standard family for short-range and medium-range communication. For long-range communication, wireless technologies such as $5 \mathrm{G}$ and LTE are preferred. However, existing technologies using both the wired and wireless medium were not designed with the SG requirements taken into consideration. SGs consist of multiple transmission points, where data are usually transmitted through multi-hop communication. With the adoption of CRNs in our proposed model, the CR HAN gateway is deployed as an essential segment that allows bi-directional flow of communication between the power providers and UCC $[57,95]$. However, the proposed model in this paper adopts $5 \mathrm{G}$ for long-range communication and then connects through the wired backhaul (e.g., fiber) to the cloud servers. As a result, this architecture facilitates multi-hop communication for SGs [96]. To resolve the issues related to cloud based architectures, this paper proposes a hybrid architecture based on fog computing and cloud computing $[97,98]$. The proposed architecture as depicted in Figure 5 is further divided into three hierarchical layers, namely smart IoT devices, fog layer, and cloud layer, as summarized below.

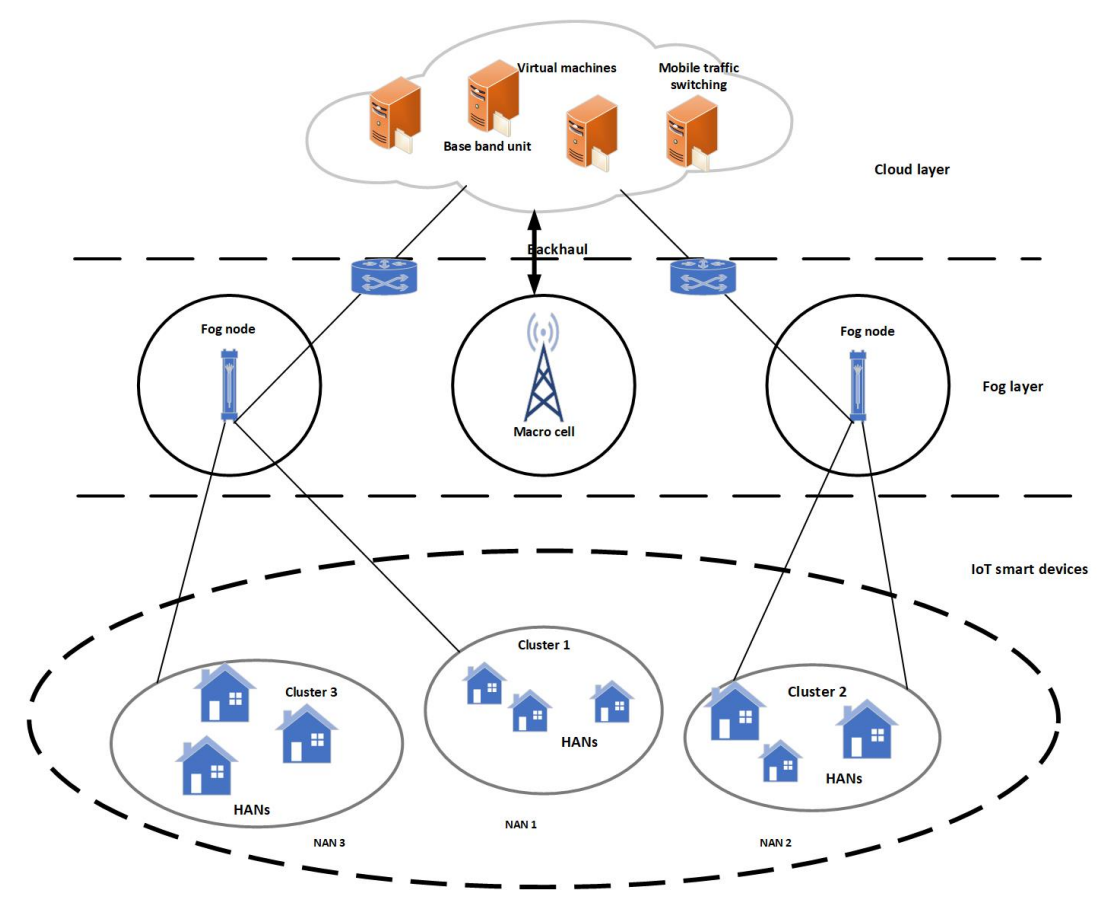

Figure 5. Smart IoT fog based architecture [99]. 


\subsection{Smart IoT Devices Layer}

This layer consists of large-scale deployment of smart IoT devices throughout the network. According to Cisco, the number of these devices is estimated to be around 50 billion, as reported in [97]. These devices include sensors, SMs, and mobile devices such as smart phones and tablets, to mention a few. These devices are envisaged to connect through $5 \mathrm{G}$ networks, which has the capacity to enhance network performance in terms of data rate, coverage, and energy efficiency. The data volume generated from the large number of devices is hard to manage by traditional cloud based architectures [99]. Thus, traditional cloud servers may not address the QoS requirements for large volumes of data in terms of latency, data rate, and response time. Fog computing can therefore be regarded as an essential technology to address the aforementioned issues. In the proposed architecture, smart IoT devices are grouped into sets of clusters to manage large data volumes. In addition, each cluster is allocated to a nearby fog node for further processing and analyses of data. Prior to clustering, fog computing utilizes the geographical information system (GIS) employed within cloud based servers to determine the location of HANs. The squared Euclidean distance $\left\|X_{i}-X_{j}\right\|^{2}$, where $j$ and $i$ denote SMs and fog nodes, respectively, can be employed for clustering [100]. The proposed clustering aims to improve the quality of communication and energy efficiency [101]. This technique further allows devices within clusters to communicate among themselves or directly through the network; this is referred to as device-to-device (D2D) or machine-to-machine (M2M) communication in the 5G context, respectively [40]. Moreover, the D2D and M2M concepts enhance interoperability within HANs. For further information on these communication technologies, readers can refer to [102]. The fog computing not only reduces the data volume in the network, but also minimizes packet losses and communication delays.

\subsection{Fog and Cloud Layer}

In Figure 5, fog nodes serve as both the gateways and edge based servers for nearby HANs. The fog nodes are distributed throughout the network to mitigate latency and packet losses by allowing communication between the HANs and cloud servers through the 5G wireless network. Due to the remote location of HANs from the cloud servers, the communication is also subject to latency issues [40]. To address this, fog computing is adopted in the form of fog nodes distributed throughout the network [99]. Furthermore, latency and packet losses are the most critical issues in this layer due to the remote location of SMs from cloud servers. As devices are clustered into sets of groups, fog nodes distinguish data packets based on their latency requirements. Latency-tolerant communication is extracted and transmitted to the cloud servers for processing, whereas latency-sensitive communication is processed in edge based servers. In future works, a framework can be developed to determine the optimal number of fog nodes, ensuring that each device in the network is covered within their range.

\section{Summary}

The work in this paper reviewed the state-of-the-art communication architectures, with the aim to find appropriate communication technologies for SG networks. This involved reviewing both existing and emerging technologies on their ability to support large-scale deployment of SGs. Furthermore, a suitable architecture was proposed based on the merits and demerits of various communication technologies. The existing technologies using both the wired and wireless medium are not equipped with the capabilities to support the distinct and stringent communication requirements of SG applications. However, 5G cellular technology has been identified as an effective and flexible technology to support SGs with high speed communication, low latency, and high reliability. To realize the benefits of $5 \mathrm{G}$ in the proposed architecture, fog computing technology has been employed to mitigate issues related to latency, security, and data congestion. Fog computing does not necessarily replace cloud servers in this framework, but serves as an extension to provide services that cloud servers may not provide. This therefore reduces packet losses as data can be transmitted to the nearby fog nodes, which can analyze and process the data instead of being transmitted to remote cloud servers 
for further processing. As it is evident that there is increasing demands for the radio spectrum in wireless communications, while the allocated spectrum is underutilized, this paper adopted CRNs with the aim to utilize unused radio spectrum bands to mitigate the issue of the scarcity of radio resources.

Author Contributions: Conceptualization, D.N.M., C.S.C. and P.N.B.; Methodology, D.N.M., C.S.C. and P.N.B.; Writing Original Draft Preparation, D.N.M.; Writing Review and Editing, D.N.M., C.S.C. and P.N.B.; Supervision, C.S.C. and P.N.B. All authors have read and agreed to the published version of the manuscript.

Funding: This research was supported by University of Johannesburg, Global Excellence and Stature 4.0 Scholarship (GES 4.0).

Conflicts of Interest: The authors declare no conflict of interest.

\section{References}

1. Xue, W; Tao, C. Research on Traditional Power Grid Enterprise Transforming to Energy Internet Enterprise. In Proceedings of the 2nd IEEE Conference Energy International Energy System Integration, Beijing, China, 20-22 October 2018; pp. 1-9.

2. Cataliotti, A.; Cipriani, G.; Cosentino, V.; Di Cara, D.; Di Dio, V.; Guaiana, S.; Panzavecchia, N.; Tinè, G. A prototypal architecture of a IEEE 21451 network for smart grid applications based on power line communications. IEEE Sens. J. 2015, 15, 2460-2467. [CrossRef]

3. Bai, X.M.; Jun-xia, M.; Zhu, N.H. Functional analysis of advanced metering infrastructure in smart grid. In Proceedings of the International Conference on Power System Technology (Powercon), Hangzhou, China, 24-28 October 2010; pp. 1-4.

4. Yu, R.; Zhong, W.; Xie, S.; Zhang, Y.; Zhang, Y. QoS differential scheduling in cognitive-radio based smart grid networks: An adaptive dynamic programming approach. IEEE Trans. Neural Netw. Learn. Syst. 2016, 27, 435-443. [CrossRef]

5. Strategic National Smart Grid Vision for the South African Electricity Supply Industry. Available online: https:/ / www.ee.co.za/wp-content/uploads/2017/12/Smart-Grid-Vision-Document-2017.pdf (accessed on 2 May 2020).

6. Ma, R.; Chen, H.H.; Huang, Y.R.; Meng, W. Smart grid communication: Its challenges and opportunities. IEEE Trans. Smart Grid 2013, 4, 36-46. [CrossRef]

7. Fang, X.; Misra, S.; Xue, G.; Yang, D. 2011. Smart grid-The new and improved power grid: A survey. IEEE Commun. Surv. Tutor. 2011, 14, 944-980. [CrossRef]

8. Kabalci, E.; Kabalci, Y. Smart Grids and Their Communication Systems; Springer: Berlin, Germany, 2019; pp. 1-609

9. Song, E.Y.; FitzPatrick, G.J.; Lee, K.B. Smart sensors and standard based interoperability in smart grids. IEEE Sens. J. 2017, 17, 7723-7730. [CrossRef]

10. Zhu, J. Communication network for smart grid interoperability. In Proceedings of the IEEE International Conference on Communication Software and Networks (ICCSN), Chengdu, China, 6-7 June 2015; pp. 260-265.

11. Ye, F.; Hu, R.Q.; Sun, H. Smart Grid Communication Infrastructures: Big Data, Cloud Computing, and Security; John Wiley \& Sons: Hoboken, NJ, USA, 2018; pp. 1-307.

12. Kazičková, T.; Buhnova, B. ICT architecture for the Smart Grid: Concept overview. In Proceedings of the Smart Cities Symposium Prague (SCSP), Prague, Czech Republic, 26-27 May 2016; pp. 1-4.

13. Garau, M.; Anedda, M.; Desogus, C.; Ghiani, E.; Murroni, M.; Celli, G. A 5G cellular technology for distributed monitoring and control in smart grid. In Proceedings of the IEEE international symposium on broadband multimedia systems and broadcasting (BMSB), Caligari, Italy, 6-7 June 2017; pp. 1-6.

14. Abdrabou, A. A Wireless Communication Architecture for Smart Grid Distribution Networks. IEEE Syst. J. 2016, 10, 251-261. [CrossRef]

15. Moslehi, K.; Kumar, R. A Reliability Perspective of the Smart Grid. IEEE Trans. Smart Grid 2010, 1, 57-64. [CrossRef]

16. Khan, A.A.; Rehmani, M.H.; Reisslein, M. Cognitive radio for smart grids: Survey of architectures, spectrum sensing mechanisms, and networking protocols. IEEE Commun. Surv. Tutor. 2016, 18, 860-898. [CrossRef] 
17. Nazari, M.H.; Grijalva, S.; Egerstedt, M. Communication-Failure-Resilient Distributed Frequency Control in Smart Grids: Part I: Architecture and Distributed Algorithms. IEEE Trans. Power Syst. 2020, 35, 1317-1326. [CrossRef]

18. Eder-Neuhauser, P.; Zseby, T.; Fabini, J. Resilience and security: A qualitative survey of urban smart grid architectures. IEEE Access 2016, 4, 839-848. [CrossRef]

19. Zhou, J.; Hu, R.Q.; Qian, Y. Scalable distributed communication architectures to support advanced metering infrastructure in smart grid. IEEE Trans. Parallel Distrib. Syst. 2012, 23, 1632-1642. [CrossRef]

20. Wang, X.; Yi, P. Security framework for wireless communications in smart distribution grid. IEEE Trans. Smart Grid 2011, 2, 809-818. [CrossRef]

21. Salvadori, F.; Gehrke, C.S.; de Oliveira, A.C.; de Campos, M.; Sausen, P.S. Smart grid infrastructure using a hybrid network architecture. IEEE Trans. Smart Grid 2013, 4, 1630-1639. [CrossRef]

22. IEEE. IEEE Guide for Smart Grid Interoperability of Energy Technology and Information Technology Operation with the Electric Power System (EPS), End-Use Applications, and Loads; IEEE Std 2030-2011; IEEE: Piscataway, NJ, USA, 2011; pp. 1-126. Available online: https://ieeexplore.ieee.org/document/6018239 (accessed on 13 March 2020).

23. Molokomme, D.N.; Chabalala, C.S.; Bokoro, P. A survey on information and communications technology infrastructure for smart grids. In Proceedings of the IEEE 2nd Wireless Africa Conference (WAC), Pretoria, South Africa, 18-20 August 2019; pp. 1-6.

24. Siozios, K.; Anagnostos, D.; Soudris, D.; Kosmatopoulos, E. IoT for Smart Grids; Springer: Cham, Switzerland, 2019; pp. 1-282.

25. Zheng, L.; Parkinson, S.; Wang, D.; Cai, L.; Crawford, C. Energy efficient communication networks design for demand response in smart grid. In Proceedings of the International Conference on Wireless Communications and Signal Processing (WCSP), Nanjing, China, 9-11 November 2011; pp. 1-6.

26. Wang, K.; Li, H.; Maharjan, S.; Zhang, Y.; Guo, S. Green energy scheduling for demand side management in the smart grid. IEEE Trans. Green Commun. Netw. 2018, 2, 596-611. [CrossRef]

27. Liu, J.; Zhao, B.; Wang, J.; Zhu, Y.; Hu, J. Application of power line communication in smart power consumption. ISPLC 2010, 303-307. [CrossRef]

28. Luhua, Z.; Zhonglin, Y.; Sitong, W.; Ruiming, Y.; Hui, Z.; Qingduo, Y. Effects of Advanced Metering Infrastructure (AMI) on Relations of Power Supply and Application in Smart Grid; IEEE: Piscataway, NJ, USA, 2010; pp. 1-5.

29. Gungor, V.C.; Sahin, D.; Kocak, T.; Ergut, S.; Buccella, C.; Cecati, C.; Hancke, G.P. A survey on smart grid potential applications and communication requirements. IEEE Trans. Ind. Inform. 2012, 9, 28-42. [CrossRef]

30. Ekanayake, J.; Liyanage, K.; Wu, J.; Yokoyama, A.; Jenkins, N. Smart Grid Technology and Applications; John Wiley \& Sons: Hoboken, NJ, USA, 2012; pp. 1-277.

31. Abdulla, G. The deployment of advanced metering infrastructure. In Proceedings of the 2015 First Workshop on Smart Grid and Renewable Energy (SGRE), Doha, Qatar, 22-23 March 2015; pp. 1-3.

32. Hongbin, Z.; Jingru, L.; Weihong, Y.; Ye, Z. The Study on Evaluating Indicator System of the Pilot Project of Smart Grid; IEEE: Piscataway, NJ, USA, 2010; pp. 1-7.

33. Song. Y.; Li, J. Research on the assessment index system for pilot projects of smart substations. IEEE PES Innov. Smart Grid Technol. 2012, 1-5. [CrossRef]

34. Simmhan, Y.; Aman, S.; Kumbhare, A.; Liu, R.; Stevens, S.; Zhou, Q.; Prasanna, V. Cloud based software platform for big data analytics in smart grids. Comput. Sci. Eng. 2013, 15, 38-47 [CrossRef]

35. Saghezchi, F.B.; Mantas, G.; Ribeiro, J.; Al-Rawi, M.; Mumtaz, S.; Rodriguez, J. Towards a secure network architecture for smart grids in 5G era. In Proceedings of the 2017 13th International Wireless Communications and Mobile Computing Conference (IWCMC), Valencia, Spain, 26-30 June 2017; pp. 121-126.

36. Chabalala, C.S.; Takawira, F. Hybrid channel assembling and power allocation for multichannel spectrum sharing wireless networks. In Proceedings of the Wireless Communications and Networking Conference (WCNC), San Francisco, CA, USA, 19-22 March 2017; pp. 1-6.

37. Huang, J.; Wang, H.; Qian, Y.; Wang, C. Priority based traffic scheduling and utility optimization for cognitive radio communication infrastructure based smart grid. IEEE Trans. Smart Grid 2013, 4, 78-86. [CrossRef]

38. Cacciapuoti, A.S.; Caleffi, M.; Marino, F.; Paura, L. Mobile smart grids: Exploiting the TV white space in urban scenarios. IEEE Access 2016, 4, 7199-7211. [CrossRef] 
39. Bansal, P.; Singh, A. Smart metering in smart grid framework: A review. In Proceedings of the 2016 Fourth International Conference on Parallel, Distributed and Grid Computing (PDGC), Waknaghat, India, 22-24 December 2016; pp. 174-176.

40. Kumari, A.; Tanwar, S.; Tyagi, S.; Kumar, N.; Obaidat, M.S.; Rodrigues, J.J. Fog computing for smart grid systems in the 5G environment: Challenges and solutions. IEEE Wirel. Commun. 2019, 26, 47-53. [CrossRef]

41. Hassebo, A.; Mohamed, A.A.; Dorsinville, R.; Ali, M.A. 5G based converged electric power grid and ict infrastructure. In Proceedings of the 2018 IEEE 5G World Forum (5GWF), Silicon Valley, CA, USA, 9-11 July 2018; pp. 33-37.

42. Erol-Kantarci, M.; Mouftah, H.T. Energy-Efficient Information and Communication Infrastructures in the Smart Grid: A Survey on Interactions and Open Issues. IEEE Commun. Surv. Tutor. 2015, 17, $179-197$. [CrossRef]

43. Vineetha, C.P.; Babu, C.A. Smart grid challenges, issues and solutions. In Proceedings of the International Conference on Intelligent Green Building and Smart Grid (IGBSG), Taipei, Taiwan, 23-25 April 2014; pp. 1-4.

44. Azizivahed, A.; Naderi, E.; Narimani, H.; Fathi, M.; Narimani, M.R. A new bi-objective approach to energy management in distribution networks with energy storage systems. IEEE Trans. Sustain. Energy 2017, 9, 56-64. [CrossRef]

45. Karandeh, R.; Lawanson, T.; Cecchi, V. Impact of operational decisions and size of battery energy storage systems on demand charge reduction. IEEE Milan PowerTech 2019, 1-6. [CrossRef]

46. Yan, Y.; Qian, Y.; Sharif, H.; Tipper, D. A Survey on Smart Grid Communication Infrastructures: Motivations, Requirements and Challenges. IEEE Commun. Surv. Tutor. 2013, 15, 5-20. [CrossRef]

47. Kansal, P.; Bose, A. Bandwidth and Latency Requirements for Smart Transmission Grid Applications. IEEE Trans. Smart Grid 2012, 3, 1344-1352. [CrossRef]

48. Ghasempour, A.; Gunther, J.H. Finding the optimal number of aggregators in machine-to-machine advanced metering infrastructure architecture of smart grid based on cost, delay, and energy consumption. In Proceedings of the 13th IEEE Annual Consumer Communications Networking Conference (CCNC), Las Vegas, NV, USA, 9-12 January 2016; pp. 960-963.

49. Kumar, P.; Lin, Y.; Bai, G.; Paverd, A.; Dong, J.S.; Martin, A. Smart grid metering networks: A survey on security, privacy and open research issues. IEEE Commun. Surv. Tutor. 2019, 21, 2886-2927. [CrossRef]

50. Kavithakumari, K.S.; Paul, P.P.; CatherineAmalaPriya, E. Advance metering infrastructure for smart grid using GSM. In Proceedings of the Third International Conference on Science Technology Engineering \& Management (ICONSTEM), Dubai, United Arab Emirates, 15-16 February 2017; pp. 619-622.

51. Zhang, Y.; Chen, M. The Development of Wirekess Networks. In Cloud Based 5G Wireless Networks; Zdonik, S., Shekar, S., Katz, J., Wu, X., Jain, L.C., Padua, D., Shen, X., Furht, B., Subrahmanian V.S., Hebert, M., Eds.; Springer Nature, Gewerbestrasse: Cham, Switzerland, 2016; pp. 1-7.

52. Mohammadi, M.; Al-Fuqaha, A. Enabling cognitive smart cities using big data and machine learning: Approaches and challenges. IEEE Commun. Mag. 2018, 56, 94-101. [CrossRef]

53. Gözde, H.; Taplamacıoğlu, M.C.; Arı, M.; Shalaf, H. 4G/LTE technology for smart grid communication infrastructure. In Proceedings of the 3rd International Istanbul Smart Grid Congress and Fair (ICSG), Istanbul, Turkey, 29-30 April 2015; pp. 1-4.

54. Dragičević, T.; Siano, P.; Prabaharan, S.R. Future generation 5G wireless networks for smart grid: A comprehensive review. Energies 2019, 12, 2140.

55. Han, Y.; Wang, J.; Zhao, Q.; Han, P. Cognitive information communication network for smart grid. In Proceedings of the 2012 IEEE International Conference on Information Science and Technology, Wuhan, China, 23-25 March 2012; pp. 847-850.

56. Yu, R.; Zhang, Y.; Gjessing, S.; Yuen, C.; Xie, S.; Guizani, M. Cognitive radio based hierarchical communications infrastructure for smart grid. IEEE Netw. 2011, 25, 6-14. [CrossRef]

57. Kumar, N. A Study on Green Energy Powered Cognitive Radio Network for Communication Network Architecture of Smart Grid. In Proceedings of the 3rd International Innovative Applications of Computational Intelligence on Power, Energy and Controls with their Impact on Humanity (CIPECH), Ghaziabad, India, 1-2 November 2018; pp. 3-7.

58. Dehalwar, V.; Kalam, A.; Zayegh, A. Infrastructure for real-time communication in smart grid. In Proceedings of the Saudi Arabia Smart Grid Conference (SASG), Jeddah, Saudi Arabia, 15-17 December 2014; pp. 1-4. 
59. Holker, D.; Brettschneider, D.; Toenjes, R.; Sonnenschein, M. Choosing communication technologies for distributed energy management in the smart grid. In Proceedings of the IEEE PES Innovative Smart Grid Technologies Conference Europe (ISGT-Europe), Torino, Italy, 26-29 September 2017; pp. 1-6.

60. Baringo, A.; Baringo, L.; Arroyo, J.M. Day-Ahead Self-Scheduling of a Virtual Power Plant in Energy and Reserve Electricity Markets Under Uncertainty. IEEE Trans. Power Syst. 2019, 34, 1881-189. [CrossRef]

61. Azizivahed, A.; Karandeh, R.; Cecchi, V.; Naderi, E.; Li, L.; Zhang, J. Multi-Area Dynamic Economic Dispatch Considering Water Consumption Minimization, Wind Generation, and Energy Storage System. In Proceedings of the IEEE Power \& Energy Society Innovative Smart Grid Technologies Conference (ISGT), Washington, DC, USA, 17-20 February 2020; pp. 1-5.

62. Eriksson, M.; Armendariz, M.; Vasilenko, O.O.; Saleem, A.; Nordström, L. Multiagent based distribution automation solution for self-healing grids. IEEE Trans. Ind. Electron. 2014, 62, 2620-2628. [CrossRef]

63. Gore, R.N.; Valsan, S.P. Wireless communication technologies for smart grid (WAMS) deployment. In Proceedings of the IEEE International Conference on Industrial Technology (ICIT), Lyon, France, 19-22 February 2018; pp. 1326-1331.

64. Moongilan, D. 5G wireless communications (60 GHz band) for smart grid-An EMC perspective. In Proceedings of the IEEE International Symposium on Electromagnetic Compatibility (EMC), Ottawa, ON, Canada, 25-29 July 2016; pp. 689-694.

65. Song, I.K.; Jung, W.W.; Kim, J.Y.; Yun, S.Y.; Choi, J.H.; Ahn, S.J. Operation schemes of smart distribution networks with distributed energy resources for loss reduction and service restoration. IEEE Trans. Smart Grid 2012, 4, 367-374. [CrossRef]

66. Rosato, A.; Panella, M.; Araneo, R.; Andreotti, A. A Neural Network Based Prediction System of Distributed Generation for the Management of Microgrids. IEEE Trans. Ind. Appl. 2019, 55, 7092-7102. [CrossRef]

67. Li, B.; Zhang, B.; Guo, J.; Yao, J. Study on Cognitive Radio Based Wireless Access Communication of Power Line and Substation Monitoring System of Smart Grid. In Proceedings of the International Conference on Computer Science and Service System, Nanjing, China, 11-13 August 2012; pp. 1146-1149.

68. Wang, Q.; Zhao, F.; Chen, T. A Base Station DTX Scheme for OFDMA Cellular Networks Powered by the Smart Grid. IEEE Access 2018, 6, 63442-63451. [CrossRef]

69. Bouabdellah, M.; El Bouanani, F.; Sofotasios, P.C.; Muhaidat, S.; Da Costa, D.B.; Mezher, K.; Ben-Azza, H.; Karagiannidis, G.K. Cooperative Energy Harvesting Cognitive Radio Networks With Spectrum Sharing and Security Constraints. IEEE Access 2019, 7, 173329-173343. [CrossRef]

70. Gungor, V.C.; Sahin, D. Cognitive Radio Networks for Smart Grid Applications: A Promising Technology to Overcome Spectrum Inefficiency. IEEE Veh. Technol. Mag. 2012 7, 41-46. [CrossRef]

71. Komninos, N.; Philippou, E.; Pitsillides, A. Survey in Smart Grid and Smart Home Security: Issues, Challenges and Countermeasures. IEEE Commun. Surv. Tutor. 2014, 16, 1933-1954. [CrossRef]

72. Jha, I.S.; Sen, S.; Agarwal, V. Advanced metering infrastructure analytics-A Case Study. In Proceedings of the 2014 Eighteenth National Power Systems Conference (NPSC), Guwahati, India, 18-20 December 2014; pp. 1-6.

73. Yu, K.; Arifuzzaman, M.; Wen, Z.; Zhang, D.; Sato, T. A Key Management Scheme for Secure Communications of Information Centric Advanced Metering Infrastructure in Smart Grid. IEEE Trans. Instrum. Meas. 2015, 64, 2072-2085.

74. Barai, G.R.; Krishnan, S.; Venkatesh, B. Smart metering and functionalities of smart meters in smart grid-A review. In Proceedings of the 2015 IEEE Electrical Power and Energy Conference (EPEC), London, ON, Canada, 26-28 October 2015; pp. 138-145.

75. Cacciapuoti, A.S.; Caleffi, M.; Marino, F.; Paura, L. Enabling Smart Grid via TV White Space Cognitive Radio. In Proceedings of the 2015 IEEE International Conference on Communication Workshop (ICCW), London, UK, 8-12 June 2015; pp. 568-572.

76. Gao, J.; Wang, J.; Wang, B.; Song, X. Cognitive radio based communication network architecture for smart grid. In Proceedings of the 2012 IEEE International Conference on Information Science and Technology, Wuhan, China, 23-25 March 2012; pp. 886-888.

77. Li, L.; Zhou, X.; Xu, H.; Li, G.Y.; Wang, D.; Soong, A. Energy-Efficient Transmission in Cognitive Radio Networks. In Proceedings of the 2010 7th IEEE Consumer Communications and Networking Conference, Las Vegas, NV, USA, 9 January 2010; pp. 1-5. 
78. Olawole, A.A.; Takawira, F.; Oyerinde, O.O. Cooperative Spectrum Sensing in Multichannel Cognitive Radio Networks with Energy Harvesting. IEEE Access 2019, 7, 84784-84802. [CrossRef]

79. Siddiqui, I.F.; Lee, S.U.; Abbas, A.; Bashir, A.K. Optimizing Lifespan and Energy Consumption by Smart Meters in Green-Cloud-Based Smart Grids. IEEE Access, 2017, 5, 20934-20945. [CrossRef]

80. Zheng, L.; Hu, Y.; Yang, C. Design and Research on Private Cloud Computing Architecture to Support Smart Grid. In Proceedings of the 2011 Third International Conference on Intelligent Human-Machine Systems and Cybernetics, Hangzhou, China, 26-27 August 2011; pp. 159-161.

81. Maheshwari, K.; Birman, K.; Wozniak, J.; Zandt, D.V. Evaluating Cloud Computing Techniques for Smart Power Grid Design Using Parallel Scripting. In Proceedings of the 2013 13th IEEE/ACM International Symposium on Cluster, Cloud, and Grid Computing, Delft, The Netherlands, 13-16 May 2013; pp. 319-326.

82. Wang, P.; Chen, X.; Sun, Z. Performance Modeling and Suitability Assessment of Data Center Based on Fog Computing in Smart Systems. IEEE Access 2018, 6, 29587-29593 [CrossRef]

83. Okay, F.Y.; Ozdemir, S. A Fog Computing Based Smart Grid Model. In Proceedings of the 2016 International Symposium on Networks, Computers and Communications (ISNCC), Yasmine Hammamet, Tunisia, 11-13 May 2016; pp. 1-6.

84. Hao, H.; Wang, Y.; Shi, Y.; Li, Z.; Wu, Y.; Li, C. IoT-G: A Low-Latency and High-Reliability Private Power Wireless Communication Architecture for Smart Grid. In Proceedings of the 2019 IEEE International Conference on Communications, Control, and Computing Technologies for Smart Grids (SmartGridComm), Beijing, China, 21-23 Octorber 2019; pp. 1-6.

85. Li, H.; Lai, L.; Zhang, W. Communication Requirement for Reliable and Secure State Estimation and Control in Smart Grid. IEEE Trans. Smart Grid 2011, 2, 476-486. [CrossRef]

86. Zaballos, A.; Vallejo, A.; Selga, J.M. Heterogeneous communication architecture for the smart grid. IEEE Netw. 2011, 25, 30-37. [CrossRef]

87. Meng, W.; Ma, R.; Chen, H. Smart grid neighborhood area networks: A survey. IEEE Netw. 2014, $28,24-32$. [CrossRef]

88. Mortaji, H.; Ow, S.H.; Moghavvemi, M.; Almurib, H.A.F. Load Shedding and Smart-Direct Load Control Using Internet of Things in Smart Grid Demand Response Management. IEEE Trans. Ind. Appl. 2017, 53, 5155-5163. [CrossRef]

89. Kuzlu, M.; Pipattanasomporn, M. Assessment of communication technologies and network requirements for different smart grid applications. In Proceedings of the IEEE PES Innovative Smart Grid Technologies Conference, Sao Paulo, Brazil, 15-17 April 2013; pp. 1-6.

90. Sun, H.; Nallanathan, A.; Tan, B.; Thompson, J.S.; Jiang, J.; Poor, H.V. Relaying technologies for smart grid communications. IEEE Wirel. Commun. 2012, 19, 52-59. [CrossRef]

91. Kalalas, C.; Thrybom, L.; Alonso-Zarate, J. Cellular Communications for Smart Grid Neighborhood Area Networks: A Survey. IEEE Access 2016, 4, 1469-1493 [CrossRef]

92. Wikström, G.; Torsner, J.; Kronander, J.; Al-Saadeh, O.; Chernogorov, F.; Bag, G.; Neander, J.; Landernäs, K.; Hovila, P. Wireless Protection of Power Grids over a 5G Network. In Proceedings of the 2019 IEEE PES GTD Grand International Conference and Exposition Asia (GTD Asia), Bangkok, Thailand, 19-23 March 2019; pp. 976-981.

93. Cosovic, M.; Tsitsimelis, A.; Vukobratovic, D.; Matamoros, J.; Anton-Haro, C. 5G Mobile Cellular Networks: Enabling Distributed State Estimation for Smart Grids. IEEE Commun. Mag. 2017, 55, 62-69. [CrossRef]

94. Sutton, G.J.; Zeng, J.; Liu, R.P.; Ni, W.; Nguyen, D.N.; Jayawickrama, B.A.; Huang, X.; Abolhasan, M.; Zhang, Z.; Dutkiewicz, E.; et al. Enabling technologies for ultra-reliable and low latency communications: From PHY and MAC layer perspectives. IEEE Commun. Surv. Tutor. 2019, 21, 2488-2524. [CrossRef]

95. Pirak, C; Sangsuwan, T.; Buayairaksa, S. Recent advances in communication technologies for smart grid application: A review. In Proceedings of the 2014 International Electrical Engineering Congress (iEECON), Pattaya City, Thailand, 19-21 March 2014; pp. 1-4.

96. Hassan, N.U.; Tushar, W.; Yuen, C.; Kerk, S.G.; Oh, S.W. Guaranteeing QoS Using Unlicensed TV White Spaces for Smart Grid Applications. IEEE Wirel. Commun. 2017, 24, 18-25. [CrossRef]

97. Islam, T.; Hashem, M.M.A. A big data management system for providing real time services using fog infrastructure. In Proceedings of the 2018 IEEE Symposium on Computer Applications \& Industrial Electronics (ISCAIE), Penang Island, Malaysia, 28-29 April 2018; pp. 85-89. 
98. Mohsenian-Rad, A.; Leon-Garcia, A. Coordination of Cloud Computing and Smart Power Grids. In Proceedings of the 2010 First IEEE International Conference on Smart Grid Communications, Gaithersburg, MD, USA, 4-6 Octorber 2010; pp. 368-372.

99. Markakis, E.; Mastorakis, G.; Mavromoustakis, C.X.; Pallis, E. Cloud and Fog Computing in 5G Mobile Networks, Emerging Advances and Applications; The Institution of Engineering and Technology: London, UK, 2017; pp. $1-425$.

100. Sinaga, K.P.; Yang, M. Unsupervised K-Means Clustering Algorithm. IEEE Access 2020, 8, 80716-80727. [CrossRef]

101. Hiew, Y.Y.; Aripin, N.M.; Din, N.M. Performance of cognitive smart grid communication in home area network. In Proceedings of the 2014 IEEE 2nd International Symposium on Telecommunication Technologies (ISTT), Langkawi, Malaysia, 24-26 November 2014; pp. 417-422.

102. Niyato, D.; Xiao, L.; Wang, P. Machine-to-machine communications for home energy management system in smart grid. IEEE Commun. Mag. 2011, 49, 53-59. [CrossRef]

(C) 2020 by the authors. Licensee MDPI, Basel, Switzerland. This article is an open access article distributed under the terms and conditions of the Creative Commons Attribution (CC BY) license (http:// creativecommons.org/licenses/by/4.0/). 